\title{
Clusters e redes de cooperação de pequenas e médias empresas: observatório europeu, caso alemão e contribuições ao caso brasileiro
}

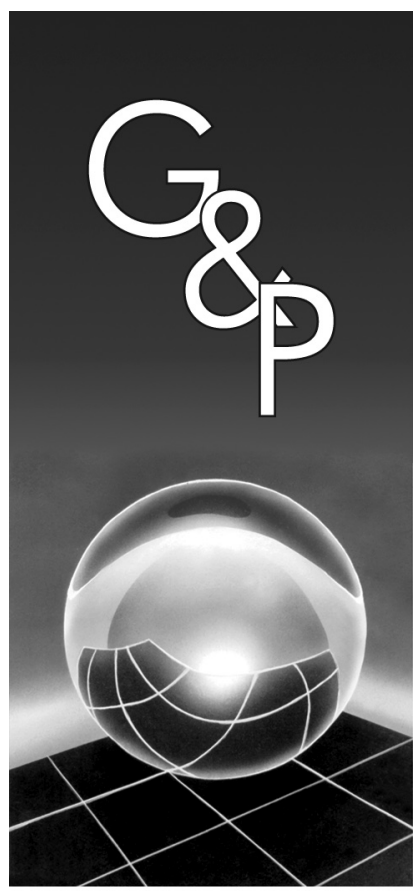

\author{
Mateus Cecílio Gerolamo \\ Luiz César Ribeiro Carpinetti \\ Timo Fleschutz \\ Günther Seliger
}

Resumo

Este trabalho apresenta os recentes estudos sobre clusters e redes de cooperação de pequenas e médias empresas na Europa, realizados pela Comissão Européia, bem como a descrição da atual estratégia de desenvolvimento de redes de competência na Alemanha (Kompetenznetze Deutschland), abordando especificamente o caso da região de Berlim e Brandemburgo. O objetivo principal é, por meio das análises dos programas europeus de desenvolvimento de clusters e redes de cooperação entre pequenas e médias empresas, verificar possibilidades de adaptação para a realidade brasileira no que se refere a arranjos produtivos locais e parques tecnológicos. Em linhas gerais, pequenas e médias empresas pertencentes a clusters na Europa têm demonstrado desempenho acima da média quando comparadas às demais empresas de seus respectivos setores. $O$ aumento da competitividade de clusters e o crescimento econômico regional estão fortemente associados ao investimento em inovação. Esse é o caso da Alemanha que incentiva o desenvolvimento de inovação por meio de suas redes de competência. A região Berlim-Brandemburgo retrata o exemplo de uma estratégia coerente de desenvolvimento local. Pode-se dizer que possíveis alternativas para o Brasil seriam o apoio mais direto do poder público, foco em inovação e definição de processos para gerenciamento das redes de cooperação.

Palavras-chave: Alemanha. Brasil. Clusters. Redes de competência. Redes de cooperação. União Européia.

\section{Introdução}

Os temas clusters e redes de cooperação têm sido pauta constante de pesquisadores e governos em vários países - desenvolvidos e em desenvolvimento. Desde os resultados bem sucedidos retratados pela Itália a partir da década de 80 e dos conceitos apresentados por Michael Porter na década de 90, a comunidade acadêmica e os governos buscam definir métodos e políticas para desenvolvimento de clusters regionais. Interessante em tais estudos e políticas é o foco em micro, pequenas e médias empresas (MPME's), pois elas desempenham um importante papel para a economia.

Em estudos realizados pela Comissão Européia instituição politicamente independente que representa e defende os interesses da Comunidade Européia - foi constatado que a maioria das pequenas e médias empresas (PME's) européias utiliza práticas de cooperação e que
PME's em clusters regionais ou redes de cooperação apresentam desempenho acima da média de sua respectiva indústria (EUROPEAN COMMISSION, 2002b; EUROPEAN COMMISSION, 2004a). A Alemanha tem se destacado em sua atual política de promoção de clusters e redes de cooperação. Atualmente o Ministério Alemão de Economia e Tecnologia (BMWi) coordena a iniciativa kompetenznetze.de, que são redes de competência focadas em inovação, consequiência de trabalhos preliminares promovidos pelo Ministério Alemão de Educação e Pesquisa (BMBF) a partir da metade da década de 90. Tais experiências internacionais podem contribuir significativamente para o aprendizado e desenvolvimento de métodos e ferramentas de gestão para clusters e redes de cooperação no Brasil com objetivo de melhorar seus desempenhos por meio de inovação e aumento de competitividade. 
Nesse contexto, este presente trabalho apresenta dois propósitos centrais. O primeiro é ilustrar a atual estratégia européia - especificamente a alemã - de desenvolvimento de clusters e redes de cooperação como forma de impulsionar a economia por meio de promoção de políticas de apoio e inovação às PME's. A outra finalidade é analisar a possibilidade de adaptação dessa experiência para a realidade brasileira, especificamente em São Paulo, como forma de incentivar o desenvolvimento por meio de políticas de investimentos em clusters e redes de cooperações de PME's, objetivando incremento em inovação e competitividade.

A seguir são apresentadas informações sobre PME's e clusters no Brasil e Europa (seção 2) bem como os principais conceitos envolvidos (seção 3). A seção 4 ilustra o Observatório Europeu enquanto a seção 5 descreve especificamente o caso alemão. Por fim, a seção 6 apresenta possíveis alternativas para o desenvolvimento de políticas voltadas à melhoria de desempenho, competitividade $\mathrm{e}$ inovação em clusters e redes de cooperação brasileiros.

\section{Clusters de pequenas e médias empresas - Brasil e União Européia}

A importância das micro, pequenas e médias empresas tanto no Brasil como na Europa pode ser claramente confirmada pelos números. O Brasil e a Europa (19 membros da União Européia) em 2003 apresentavam estatísticas similares quanto à participação das MPME's em relação ao número total de empresas, ou seja, $99 \%$. A porcentagem de empregados nas MPME's significava mais de $65 \%$ do total. Entretanto a participação das MPME's nas exportações representava 39\% do total na Europa enquanto que $21 \%$ no Brasil. Quanto aos problemas identificados, no Brasil pelo menos $50 \%$ das pequenas empresas apresentava um ciclo de vida inferior a cinco anos devido a questões como falta de capital de giro e alta incidência de impostos. Além disso, existiam em 1997 aproximadamente 9,5 milhões de empresas informais por todo o país, responsáveis pelo emprego de, pelo menos, 12 milhões de pessoas. Dentre as restrições identificadas no caso europeu, destacavam-se: i) falta de poder de compra por parte de seus clientes, a qual é ainda mais crítica para as microempresas; ii) escassez de mão-de-obra qualificada, a qual é mais realçada pelas médias empresas; iii) dificuldade de acesso a financiamentos; e iv) regulamentações administrativas. Os últimos dois itens com mais intensidade nas pequenas empresas. (SEBRAE, 2005; EUROPEAN COMMISSION, 2004b).

O Observatório realizado na Europa também realça que o crescimento econômico está positivamente associado a uma importante função das PME's, que apresenta três impactos externos sobre a economia como um todo (EUROPEAN COMMISSION, 2004b): a) PME's servem como um veículo de transferência de conhecimentos e que podem se tornar acessíveis por grandes empresas por meio de transferência ou aquisição de tecnologias;

b) PME's aumentam a competição nos mercados, por meio de novas idéias e capital humano incorporado ao conhecimento dos trabalhadores; $\mathrm{e}$

c) PME's aumentam a diversidade nos mercados, que podem se desenvolver e gerar aumento de produtividade nas empresas.

A Alemanha em específico apresenta dados referentes à média européia. Comprovando a sua importância econômica no Mercado Europeu, os dados ainda mostram que a Alemanha representava $16 \%$ do total de MPME's e empregava cerca de $21 \%$ do total de pessoas ocupadas em todas as MPME's na Comunidade Européia entre seus 19 membros em 2003 (EUROPEAN COMMISSION, 2004b).

Quanto aos clusters no Brasil, Puga (2003) identificou 193 aglomerações industriais no Brasil compostas por aproximadamente 77 mil empresas responsáveis por cerca de 680 mil empregos, o que corresponde a mais do que $3 \%$ dos empregos no país, e por exportações no valor de US\$ 3,8 bilhões, que corresponde a cerca de $6 \%$ do total de exportações. Entretanto, de acordo com o Ministério Brasileiro do Desenvolvimento, Indústria e Comércio Exterior (2006), existem quase mil clusters de PME's no Brasil considerando os setores de indústria, agronegócios, comércio e turismo. Por outro lado, de acordo com Quandt (2004), apesar da existência de muitos clusters no Brasil, a maioria apresenta baixos índices de inovação tecnológica, existindo apenas alguns clusters emergentes que reúnem empresas de base tecnológica. Segundo Carvalho et al. (2000), as indústrias de base tecnológica no Brasil encontram-se concentradas nas regiões sul e sudeste, situando-se no Estado de São Paulo quase $30 \%$ dos Parques Tecnológicos existentes no país. O Governador do Estado de São Paulo instituiu em 2006 o Sistema Paulista de Parques Tecnológicos, que tem por objetivo fomentar, impulsionar e apoiar iniciativas de criação e implantação dos parques. Para tal foram destinados 9 milhões de reais para a concretização dos três primeiros parques, ou seja, Campinas, São Carlos e São José dos Campos. Para Quandt (2004) é evidente o papel da inovação para ampliar as oportunidades de ganhos econômicos e sociais das cidades e regiões, reforçando ainda mais a importância econômica e social que as PME's envolvidas em clusters ou redes de cooperação têm frente ao cenário atual da necessidade de inovação e incentivo ao crescimento e desenvolvimento do país.

Quanto à existência de clusters na Europa, Van der Linde (2003), em seu estudo sobre a demografia de clusters em países desenvolvidos (25) e em desenvolvimento (24), identificou 457 clusters na Europa, em 15 países membros. Já os dados da Comissão Européia 
(EUROPEAN COMMISSION, 2002b) apresentam cerca de 900 clusters, porém com base em estudos individuais de cada um dos 10 países dentre os 19 países membros. Apesar da Comissão Européia (EUROPEAN COMMISSION, 2002b) não ter identificado estudos de mapeamento de clusters regionais na Alemanha, existem até o presente momento cerca de 130 redes de competência (kompetenznetze), compreendendo em alguns casos clusters, em outros, partes de um cluster.

Embora a comparação entre clusters seja difícil pelo fato deles não apresentarem as mesmas características, já é possível notar que clusters regionais europeus apresentam melhor desempenho em aspectos gerais quando comparados com a média nacional nas respectivas indústrias (EUROPEAN COMMISSION, 2002b). Por outro lado, percebe-se que há ainda uma falta de informações que permitam melhores análises dos clusters europeus (KETELS, 2004). Antes, porém, de entrar em mais detalhes sobre os resultados dos observatórios europeus de PME's, clusters e redes de cooperação, e o caso das redes de competência alemãs, o tópico a seguir aborda os principais conceitos com o objetivo de embasar a discussão subseqüente.

\section{Clusters e redes de cooperação - conceitos}

\subsection{Definições}

Como um dos autores atualmente mais citados, Porter (1998) define clusters como uma concentração geográfica de empresas interconectadas, fornecedores e prestadores de serviço especializados, firmas em indústrias relacionadas, entre outras entidades de interesse pertencentes a um setor industrial específico onde há concorrência, mas também práticas de cooperação. Exemplos de tais entidades de interesse são: universidades, centros de pesquisa, agência de normatização, associação de indústrias, poder público, entre outras.

Entretanto, o que torna os clusters potencialmente benéficos para a competitividade de PME's é o fato de existirem oportunidades para se obter eficiências coletivas, derivadas de economias externas e desenvolvimento de ações conjuntas (SCHMITZ, 1999). O conceito de economias externas, primeiramente desenvolvido por Alfred Marshall em seu clássico livro "Princípios de Economia", publicado em 1890, envolve vantagens como fornecedores especializados de matéria-prima, equipamentos e serviços específicos; especialização de mão-de-obra qualificada; e disseminação de novos conhecimentos e tecnologia (KRUGMAN, 1991).

Agregando mais elementos à teoria de aglomerações industriais, Schmitz (1999) e Schmitz e Nadvi (1999) argumentam que os resultados de economias externas não são suficientes para explicar o desenvolvimento e o crescimento de clusters. Apesar das economias externas ainda serem vistas como importante elemento, esses autores introduzem o conceito de ações conjuntas para explicar os benefícios dos clusters. Tais ações podem ser na forma de cooperação horizontal, vertical ou ainda bilateral ou multilateral. Esses autores definem então eficiência coletiva como a vantagem competitiva derivada de economias externas locais somadas ao desenvolvimento de deliberadas ações conjuntas.

De acordo com Andriani et al. (2005), clusters possuem as seguintes características:

a) uma massa crítica de empresas e instituições localizadas em uma mesma área geográfica e especializadas em um conjunto de atividades econômicas interdependentes;

b) firmas que se especializam em diferentes aspectos da cadeia de valor; por causa de sua complementaridade, as empresas são integradas por uma divisão externa de trabalho resultando em redes de relacionamento de entradas e saídas (input-output links);

c) as empresas são incorporadas (embedded) em uma densa rede de interdependência (social e cultural) não usual que gera oportunidades para aprendizado mútuo e eleva o nível de cooperação;

d) um processo equilibrado de tomada de decisão, o qual não apresenta características claramente hierárquicas (isto significa uma estrutura de governança horizontal); e

e) institutional thickening, que é a existência de instituições e organizações públicas e privadas capazes de dar suporte para o crescimento dos clusters.

Clusters e redes de cooperação têm sido vistos por muitos, acadêmicos e autoridades públicas, como instrumentos que podem estimular a competitividade de PME's, regiões e países por meio de inovação. Essa é, entretanto, uma árdua tarefa considerando o atual ambiente competitivo, no qual a inovação ocorre por meio de interações não seqüienciais entre diferentes companhias, universidades e instituições de pesquisa, ou seja, um modelo um pouco diferente do modelo tradicional, no qual centros de pesquisa e desenvolvimento transformam pesquisa básica advinda da universidade em produtos e processos aplicados (KETELS, 2004).

Nesse contexto de inovação via interação entre variados elementos, percebe-se, portanto, a importância da palavra-chave "cooperação". De acordo com a definição da Comissão Européia (EUROPEAN COMMISSION, 2004a), cooperação pode ser entendida como a relação entre parceiros independentes que combinam seus esforços e recursos num processo conjunto de criação de valor. Cooperação é, portanto, a integração entre os parceiros sendo esses integrados por uma estrutura que promove a parceria. A cooperação entre vários parceiros 
pode levar à construção de uma rede e o trabalho em rede tem potencial de adquirir sinergia. Dessa forma, para que haja criação de valor na rede, a divisão de conhecimento e a troca de idéias são de fundamental importância. E isso depende da construção de confiança entre os parceiros.

Dada a variedade de conceitos para o termo cluster, a Comissão Européia (EUROPEAN COMMISSION, 2002b) salienta a necessidade de se trabalhar com definições mais precisas para servirem como base para decisões políticas. Assim, é assumido, de forma simplificada, que clusters são concentrações geográficas de empresas interconectadas. A Comissão Européia utiliza então o termo regional innovation systems ou sistemas regionais de inovação, que significam clusters mais instituições de suporte com o objetivo de desenvolver e difundir o conhecimento. Entretanto, antes do cluster se constituir em um sistema de inovação, as empresas devem primeiro formar redes regionais de inovação envolvendo cooperação mais organizada e formal estimulada pela confiança, por normas e princípios, os quais encorajam as empresas a executarem atividades de inovação.

\subsection{Fases de desenvolvimento}

Adicionalmente, deve-se considerar que os clusters apresentam graus de desenvolvimento diferentes e essa questão deve ser sempre levada em conta quando forem realizadas propostas de intervenção. Nesse sentido, Andriani et al. (2005) discutem duas importantes questões: como clusters emergem? E o que acontece quando clusters embrionários se tornam sistemas localizados de produção mais desenvolvidos? Quanto à primeira questão, os autores identificaram combinações específicas de condições que possam favorecer a emergência de clusters: a existência de uma força de trabalho especializada; oportunidades tecnológicas ou de mercado; e demanda e acesso a mercados. Com relação à segunda questão, uma vez que os clusters embrionários se desenvolvem, modelos mais estáveis de interações entre diferentes stakeholders começam a surgir.

Entretanto é importante salientar que, em certas circunstâncias, clusters podem tornar-se um empecilho para o desenvolvimento de seus membros e, em situações extremas, levar até mesmo ao declínio econômico de suas regiões (EUROPEAN COMMISSION, 2002c). Toda a estrutura montada para o funcionamento do cluster pode tornar-se rígida a ponto de impedir mudanças necessárias ao longo do tempo. Isso é denominado como "especialização rígida" que impede o surgimento de novas idéias e pode ser uma ameaça ao desenvolvimento do cluster e da região (EUROPEAN COMMISSION, 2002b). A Comissão Européia define de forma mais clara seis estágios para abordar um modelo de desenvolvimento de clusters (Tabela 1).

Pesquisas e políticas direcionadas a clusters devem, portanto, incentivar um processo contínuo de melhoria e inovação para evitar que possíveis barreiras levem ao declínio de desempenho do cluster e possivelmente ao fracasso econômico de sua respectiva região. O ideal é pensar em termos de melhoria contínua de desempenho, mas é necessário nesse ponto responder à seguinte questão: o que faz a diferença para que um cluster apresente um comportamento de desenvolvimento contínuo?

\subsection{Gestão de clusters e redes de cooperação}

Certo grau de coordenação é importante para que um cluster regional deixe de ser apenas um aglomerado de empresas e torne-se uma rede ou um sistema regional de inovação por meio de projetos de cooperação. Esse papel pode ser assumido por meio da criação e manutenção de institutional thickness, ou seja, organizações formais com alto nível de interação que podem dar suporte às empresas de variadas maneiras; ou instituições sociais que promovam uma densa e informal colaboração entre as pessoas nas empresas e organizações. Elas são importantes para o crescimento e sucesso de clusters, pois estimulam a capacidade de inovação como uma propriedade da região (EUROPEAN COMMISSION, 2002b)

De acordo com Andriani et al. (2005), as agências de desenvolvimento regionais (ADR's) são bons exemplos para atuação em necessidades específicas, avaliando opções políticas e trabalhando com as empresas e outras organizações para ajudar a sustentar os clusters competitivos. Segundo o professor Jörg Sidow, presidente do

Tabela 1. Estágios de desenvolvimento de clusters regionais: um modelo conceitual. (EUROPEAN COMMISSION, 2002b, p. 15).

\begin{tabular}{|c|c|}
\hline Estágios & Descrição \\
\hline 1 & $\begin{array}{l}\text { Formação de firmas pioneiras freqüentemente embasadas em conhecimento local específico, seguidas por novas firmas } \\
\text { spin-off. }\end{array}$ \\
\hline 2 & $\begin{array}{l}\text { Criação de um conjunto de fornecedores e firmas de prestação de serviços especializados e oferta de mão-de-obra } \\
\text { especializada. }\end{array}$ \\
\hline 3 & Formação de novas organizações que servem às firmas do cluster. \\
\hline 4 & Atração de firmas externas e trabalhadores qualificados, e boas oportunidades para o surgimento de novas firmas locais. \\
\hline 5 & $\begin{array}{l}\text { Criação de ativos intangíveis (não quantificáveis) que estimulam a circulação informal de informação e o conhecimento } \\
\text { no cluster. }\end{array}$ \\
\hline 6 & $\begin{array}{l}\text { Um período de declínio pode ocorrer no cluster em virtude de situações lock-in, ou seja, ele pode adquirir uma estrutura } \\
\text { rígida e se tornar inflexível a mudanças e novas idéias. }\end{array}$ \\
\hline
\end{tabular}


conselho de assessoria do Programa Redes de Competência Alemãs, enquanto os gerentes das organizações tradicionais têm se tornado cada vez mais profissionais, os coordenadores de redes de cooperação e clusters (quando eles existem) ainda enfrentam uma série de desafios gerenciais. (BUNDESMINISTERIUM FÜR WIRTSCHAFT UND TECHNOLOGIE, 2006).

Esses argumentos anteriormente mencionados são reforçados por uma das recomendações sugeridas pelo grupo de especialistas da Comissão Européia (EUROPEAN COMMISSION, 2002c) que afirmam que mais esforços são necessários para, dentre outras razões, implementar estruturas para gerenciamento de clusters. Andriani et al. (2005) consideram também que, dentre outras questões importantes que ainda precisam ser mais bem analisadas, é preciso entender melhor quais tipos de infra-estruturas são necessários e, talvez ainda mais importante, como induzir pequenas companhias a fazerem bom uso de tais infra-estruturas.

Apesar do grande número de trabalhos publicados, percebe-se que ainda são poucas as contribuições que apresentam metodologias e ferramentas de gestão, as quais os clusters necessitam para aumentar seu grau de coordenação. Relembrando um dos pontos iniciais deste tópico, onde Schmitz (1999) e Schmitz e Nadvi (1999) realçam a importância não apenas de economias externas, mas também de ações deliberadas para se chegar à eficiência coletiva, podemos concluir que tais instituições formais ou sociais que apresentem uma estrutura de gestão do cluster são fundamentais para direcioná-lo à melhoria contínua de desempenho e inovação reduzindo, portanto, a probabilidade de entrar em declínio em função de uma estrutura rígida e inflexível a mudanças e inovações.

\subsection{A importância de políticas de apoio a clusters e redes de cooperação}

Clusters regionais deveriam formar um importante grupo-alvo de políticas industriais e de inovação, pois podem ser uma eficiente maneira de se criar trabalho e riqueza em regiões. Mais do que isso, eles podem servir como alavanca para o desenvolvimento sustentável, ou seja, não apenas para o desenvolvimento econômico, mas também social e ambiental (EUROPEAN COMMISSION, 2002c). Para a Comissão Européia (EUROPEAN COMMISSION, 2004a), políticas direcionadas a um cluster específico serão mais efetivas se desenvolvidas em nível local ou regional, pois clusters são muitos diferentes e necessitam de ações individuais. As políticas devem ser formuladas mostrando os benefícios que as empresas/os empresários terão ao engajarem-se em projetos de cooperação e ainda devem tentar diminuir ao máximo as principais barreiras existentes à prática de cooperação.
A idéia de aglomeração realça a função de autoridades públicas como um facilitador entre empresas e entre empresas e infra-estrutura de conhecimento. De acordo com o parecer da Comissão Européia (EUROPEAN COMMISSION, 2002b), a função do setor público deveria ser a de incentivar o processo de planejamento estratégico. O setor público, em parceria com o setor privado, pode criar condições de suporte e impulso para novas atividades econômicas e assim melhorar a capacidade regional de inovação. $\mathrm{O}$ papel das autoridades públicas deve ser então de facilitador no suporte à política de clusters. Entende-se, portanto, como importante o estabelecimento de organizações especializadas (institutional thickness) que podem até ser instituídas pelas próprias empresas, entretanto com suporte público. Ainda, as políticas de apoio podem incluir estímulos para que os clusters melhorem seu desempenho e tornem-se redes inovativas ou sistemas inovativos locais (EUROPEAN COMMISSION, 2002b).

De acordo com Ketels (2004), em sua análise sobre os clusters europeus, para se fazer políticas mais efetivas, pontos importantes ainda devem ser considerados: i) mais informações sobre o atual status dos clusters devem ser levantadas; ii) as especificidades entre diferentes clusters e diferentes regiões devem ser consideradas em políticas de desenvolvimento de clusters; iii) clusters devem ser vistos como parte de uma agenda mais abrangente de competitividade, ou seja, esforços para o desenvolvimento econômico de clusters e regiões precisam ser mais bem integrados; e iv) clusters podem ser vistos como uma oportunidade para se definir as regras dos setores privados e públicos na política econômica, cada um assumindo a responsabilidade por suas específicas áreas de competência no ambiente de negócios.

Ainda que muito mais pudesse ser discutido no âmbito conceitual, os elementos anteriores fornecem uma sólida base para a análise dos observatórios realizados na Europa, e uma melhor compreensão do caso alemão de desenvolvimento de redes de competência regionais, descrito a seguir.

\section{Observatório europeu de pequenas e médias empresas, redes de cooperação e clusters}

\subsection{Pequenas e médias empresas e cooperação na Europa}

A Comissão Européia encontrou as seguintes principais conclusões relacionadas ao processo de cooperação entre PME's (EUROPEAN COMMISSION, 2004a):

a) ao todo, mais da metade das PME's apresentam práticas de cooperação (formais ou informais) com pelo menos outra empresa, destacando-se as PME's 
da Finlândia, Noruega, Islândia, Dinamarca e Itália; a cooperação informal é mais comum do que a formal;

b) possíveis razões para o uso de práticas de cooperação podem ser explicadas em função de uma cultura comum e uma base social, como observado nos países nórdicos, ou iniciativas políticas com objetivo de estimular a cooperação entre PME's como ocorrido na Itália; quanto às motivações destacam-se: acesso a novos e maiores mercados, mais amplo fornecimento de produtos, e acesso a know-how e tecnologia, entre outros;

c) há uma preferência por redes com número limitado de parceiros; mais da metade das firmas que cooperam têm contato com seus parceiros, pelo menos, uma vez por semana; a cooperação entre as PME's européias é caracterizada por relações estáveis e parcerias com duração superior a cinco anos;

d) as principais barreiras que prejudicam o processo de cooperação são: desejo de manter independência, falta de informação sobre com quem cooperar, ausência de interesse em abrir importantes informações para outras PME's, entre outras;

e) quanto ao desempenho, oito em cada dez PME's pesquisadas reportaram que o processo de cooperação com outras empresas melhorou sua força competitiva;

f) micro e pequenas empresas cooperam objetivando conquistar novos e maiores mercados, enquanto as médias buscam redução de custos; a cooperação informal é mais praticada nas micro e pequenas; as micro apresentam menos parceiros do que as pequenas que, por sua vez, apresentam menos parceiros do que as médias; micro e pequenas empresas fazem contatos várias vezes por semana enquanto médias empresas, pelo menos, uma vez por mês; tanto micro, pequenas e médias empresas atestam que a principal barreira para o processo de cooperação é o desejo de manter independência; e

g) quanto ao desenvolvimento de políticas de apoio à cooperação entre PME's foi observado que um dos instrumentos usados para esse propósito é o incentivo ao desenvolvimento de clusters regionais; o Observatório de PME's Européias 2002 (EUROPEAN COMMISSION, 2002b) estudou e comparou 34 clusters regionais em toda a Europa, conforme descreve o tópico seguinte.

\subsection{Clusters regionais europeus}

Nesse estudo (EUROPEAN COMMISSION, 2002b), foram examinados 17 clusters tradicionais e 17 clusters de base tecnológica. Em resumo, os resultados do estudo de clusters regionais europeus mostram que os clusters apresentam-se em crescimento tanto em relação ao número de empresas quanto ao número de empregados. Eles são dominados por um número cada vez maior de PME's, mas ao mesmo tempo empresas multinacionais vêm também aumentando sua presença. Os clusters estão também incorporados em redes globais, entretanto um número de atividades ao longo da cadeia de valor das principais firmas do cluster situa-se dentro das fronteiras geográficas do cluster e cada vez mais as firmas têm encontrado seus parceiros de pesquisa e desenvolvimento dentro do cluster.

Relações de longo prazo e coalizões temporárias têm se tornado notoriamente mais comum nos últimos dez anos. Os resultados também mostram que as firmas nos clusters cooperam intensivamente com fornecedores de serviços, instituições de pesquisa e desenvolvimento e autoridades públicas. Nesse ponto, destaca-se a importância de organizações formais como colaboradores importantes no processo de desenvolvimento, conforme mencionado anteriormente em institutional thickness. Existe uma evidência na pesquisa de que tais instituições estão aumentando sua importância no processo de coordenação das atividades do cluster. Além disso, os clusters são muito diferentes com relação à posição competitiva e às atividades de inovação. Mudanças e inovações na organização do processo produtivo são importantes e mais direcionadas a clusters de base tecnológica. Inovações em métodos de marketing e distribuição são também importantes, porém em clusters tradicionais (EUROPEAN COMMISSION, 2002b).

Para aquelas empresas que buscam aquisição de know-how, novas tecnologias e, por meio disso, inovação, revela-se como um importante fator a cooperação, não apenas com centros de conhecimento, mas também com relação ao estabelecimento de redes num sentido mais amplo. A existência de redes possibilita a disseminação de conhecimento e a divisão de custos e riscos para as PME's (EUROPEAN COMMISSION, 2002a).

Entretanto, barreiras conforme as citadas na seção anterior ainda prejudicam a promoção de práticas cooperativas entre PME's. Assim, para que as barreiras à cooperação sejam eliminadas ou reduzidas, políticas de desenvolvimento devem considerá-las tanto em nível nacional como, ainda mais importante, em nível regional. O tópico seguinte aborda o tema política de apoio a clusters e redes de cooperação, ilustrando algumas políticas observadas nos clusters europeus identificadas pelo estudo da Comissão Européia.

\subsection{Políticas observadas nos clusters europeus}

Entre os 34 clusters estudados (EUROPEAN COMMISSION, 2002b), 28 apresentam organizações especializadas, sendo muito mais comum nos clusters de base tecnológica do que nos clusters tradicionais. As políticas de apoio mais importantes identificadas entre os clusters europeus estudados, em ordem de importância, são: i) infra-estrutura de suporte (física e de conheci- 
mento e serviços específicos ou centros de tecnologia); ii) suporte para treinamento e pesquisa; e iii) suporte financeiro para projetos entre as firmas; entre outras.

O Ministério Português do Ambiente, do Ordenamento do Território e do Desenvolvimento Regional (2006) destaca como essencial o uso de metodologias para uma avaliação rigorosa dos apoios a conceder, evitando dessa forma a dispersão dos recursos. Em todos os casos estudados pelo governo português na França, Alemanha, Itália e Espanha, a constituição dos sistemas de inovação regional sustenta-se na elaboração prévia de um projeto detalhado, no qual devem conter todos os elementos necessários para a configuração do sistema e para a análise de sua viabilidade. Tais elementos são: áreas de pesquisa, áreas de produção, atores envolvidos e suas funções e responsabilidades, recursos humanos e materiais, objetivos de desenvolvimento, indicadores de medida dos objetivos, formas de avaliação, cálculos financeiros e estruturas de governança.

Embora a abordagem seja diferenciada caso a caso, políticas nacionais observadas na União Européia apresentam algumas características gerais (EUROPEAN COMMISSION, 2002b): promovem o desenvolvimento econômico e mudanças estruturais; abordam a capacidade regional de inovação; baseiam-se em cooperação e networking; enfatizam a integração entre firmas e infraestruturas tecnológicas de educação e instituições de pesquisa e desenvolvimento locais; tentam trazer novas tecnologias para redes regionais de PME's; enfatizam a função de organizações públicas ou semipúblicas para encorajar o relacionamento interfirmas e projetos conjuntos; e indicam a importância da necessidade de melhorar a capacidade de inovação e a gestão do conhecimento entre as PME's.

Dessa forma, no que se refere à União Européia, Ketels (2004) entende que a Comissão Européia vem assumindo um importante papel para o desenvolvimento de clusters na Europa por meio de fornecimento de melhores dados sobre clusters, articulação de pesquisas conjuntas entre diferentes instituições com foco em questões relativas à realidade européia, e suporte às iniciativas de clusters regionais.

A seção a seguir descreve o recente caso alemão de desenvolvimento de seus clusters e redes de cooperação focando em redes de competência e inovação.

\section{A Alemanha e as redes de cooperação entre pequenas e médias empresas}

\subsection{Antecedentes}

Em seu livro sobre a dinâmica do capitalismo industrial, Chandler (1990) aborda historicamente a Alemanha como um caso de capitalismo gerencial cooperativo. A coletividade das indústrias revelou-se mais forte na
Alemanha do que em países como Estados Unidos ou Grã-Bretanha, apresentando um relacionamento interfirma e intrafirma muito mais intenso. Investimentos de base fizeram com que a Alemanha, mesmo sendo muito afetada pela $1^{a}$ Grande Guerra, conseguisse recuperação entre 1924 e 1930 por meio de suas empresas líderes. Nesse contexto surgiram fortes relações de cooperação entre empresas verticalmente integradas com o objetivo de se posicionar nos mercados estrangeiros. Dessa forma a Alemanha, que foi muito mais abalada com a $1^{\text {a }}$ Guerra, conseguiu recuperar seu poder frente ao mercado mundial. Desenvolvendo suas habilidades organizacionais, os empreendedores alemães conseguiram reforçar as relações de cooperação interfirmas, fator essencial para a recuperação das grandes indústrias alemãs.

Fato marcante na Alemanha e decorrente dos investimentos ocorridos ao longo do processo histórico de desenvolvimento do país é a presença de um amplo número de instituições de pesquisa. Isso possibilita às empresas assumirem um caráter não apenas baseado na indústria e produção, mas também baseado em pesquisa e desenvolvimento. Destacam-se universidades, escolas técnicas, institutos nacionais de pesquisa como, por exemplo: Max Planck, Fraunhofer, Helmholtz, Leibniz; e outros centros regionais de pesquisa como, por exemplo, a Fundação de Tecnologia de Berlim (TSB); instituições de apoio à pesquisa básica como a Fundação Alemã de Apoio à Pesquisa (DFG) e pesquisa aplicada como a Federação Alemã de Pesquisa Industrial (AiF), entre outros.

\subsection{O surgimento de uma política nacional de apoio a clusters na Alemanha}

De acordo com o relatório de clusters regionais apresentado pela Comissão Européia (EUROPEAN COMMISSION, 2002c), até o final da década de 90 a abordagem alemã para política de desenvolvimento tecnológico raramente considerou o aspecto regional. Tais políticas eram mais utilizadas para compensar disparidades regionais (como o caso de regiões pertencentes à antiga Alemanha Oriental) ao invés de fortalecer clusters regionais com propósito de melhoria de competitividade em nível internacional. A falta de um mapa compreensivo de clusters alemães e os crescentes argumentos científicos focando em regiões como fator-chave no processo de mudança tecnológica incentivaram o governo alemão a iniciar o programa BioRegio, no qual as regiões alemãs com foco em biotecnologia deveriam competir por fundos públicos. Percebendo uma lacuna tecnológica nessa indústria quando comparada aos Estados Unidos ou Reino Unido, o governo alemão considerou os resultados bem sucedidos de políticas de clusters realizados por outros países. O programa BioRegio representa, dessa forma, uma notável mudança na política de desenvolvimento tecnológico alemã e foi seguido por outros 
programas como EXIST e InnoRegio acompanhados por instrumentos políticos com foco em desenvolvimento regional (BUNDESMINISTERIUM FÜR BILDUNG UND FORSCHUNG, 2002; 2004 e 2005). Atualmente, a Alemanha conta com várias possibilidades de suporte para clusters ou para formação de redes de pequenas e médias empresas.

EmboraorelatóriodaComissãoEuropéia(EUROPEAN COMMISSION, 2002b) afirme que a Alemanha não apresente instrumentos políticos para o desenvolvimento de clusters, atualmente pode-se entender, conforme constatado pelos exemplos anteriores, que a Alemanha vem apresentando nos últimos dez anos um notável foco para o desenvolvimento de PME's e redes de cooperação. Em nível nacional, destaca-se a iniciativa Redes de Competência Alemãs, iniciada em 1999, abrangendo esse aspecto de direcionamento para o desenvolvimento de clusters e redes de cooperação, conforme aborda o texto a seguir.

\subsection{Desenvolvimento de redes de competência}

Atualmente, o governo alemão está promovendo o fortalecimento nacional por meio de uma gama de atividades. Uma das mais importantes direcionadas às PME's é a iniciativa "Redes de Competência Alemãs" conhecida também como Kompetenznetze Deutschland ou ainda kompetenznetze.de. Esta é uma iniciativa encampada pelo Ministério Alemão de Economia e Tecnologia (BMWi). A iniciativa é fruto do resultado de uma intensiva discussão sobre o tema Clusters Regionais de Inovação, realizada pelo Ministério Alemão de Educação e Pesquisa (BMBF) ao longo dos anos 90. A Kompetenznetze Deutschland foi então iniciada no ano de 1999 com 20 redes de competência que propuseram projetos iniciais com duração de dois anos encaminhados para a Associação Alemã de Engenharia (VDI-TZ). A partir de então o Departamento de Tecnologia do BMBF realizou sucessivos desdobramentos em direção a implementações de projetos com abordagens de centros de competência.

Atualmente a iniciativa kompetenznetze.de possui mais de 130 redes de competência em 18 campos de inovação em mais de 30 regiões na Alemanha. A Figura 1 apresenta o mapa da Alemanha com 37 regiões e os atuais campos de inovação, focos da iniciativa. Essas regiões não são caracterizadas por fronteiras administrativas, mas sim pela área de atividade da rede e a localização de seus membros. Freqüentemente, essas regiões de inovação compreendem mais de uma rede de competência. No mapa a seguir, as regiões são representadas por círculos, que por sua vez são maiores onde existem mais redes de competência residentes.

Para que um aglomerado ou uma rede de cooperação faça parte da iniciativa kompetenznetze.de é preciso que sejam respeitados alguns critérios de admissão (VELLING, 2006), conforme descreve a Tabela 2.

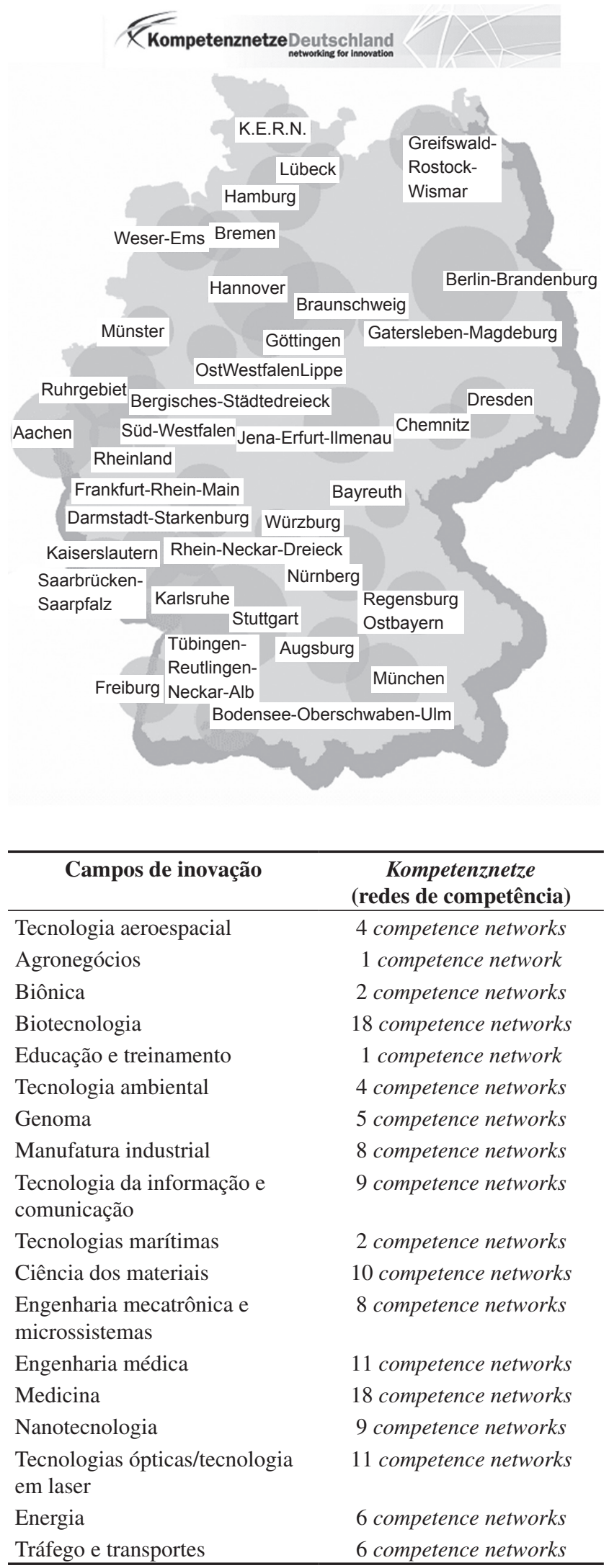

Figura 1. Regiões e campos de inovação na Alemanha. (KOMPETENZNETZE DEUTSCHLAND: NETWORKING FOR INNOVATION, 2008). 
De acordo com Dr. Johannes Velling (VELLING, 2006), diretor da Unidade Fundação de Tecnologia, Financiamento, cluster de competência do BMWi, as kompetenznetze são algumas vezes idênticas a clusters e compreendem freqüentemente partes de um cluster. Para ele, os critérios 1,2 e 4 anteriormente definidos compreendem os critérios de clusters definidos por Michael E. Porter. Dessa forma, de posse desses critérios e características, a Figura 2 apresenta um modelo com a proposta do que deve ser uma estrutura ideal para o funcionamento de uma rede de competência.

Assim, os membros das redes enxergam benefícios em participar da iniciativa por apresentar uma diferenciação comparada a outras redes e clusters individuais, pois podem utilizar o nome kompetenznetze.de como estratégia de marketing e uma espécie de selo de qualidade - um clube das melhores redes de inovação da Alemanha (ESTERMANN, 2006). Isso permite uma maior visibilidade para as PME's que compõem as redes de competência a fim de buscar mais parceiros para cooperação e investidores tanto nacionais como estrangeiros, articulando com mais força pedidos de investimentos. Além disso, a iniciativa em nível nacional ainda permite o investimento em marketing com apoio do escritório central da iniciativa, consultorias, intermediação de contatos e uma troca de informações e aprendizado com outras redes de competência por meio de benchmarking cooperativo (VELLING, 2006).

De acordo com BMWi, para os anos de 2006 a 2009, serão disponibilizados 6 bilhões de euros para pesquisa e desenvolvimento, dos quais 600 milhões já foram investidos em 2006. O BMWi tem identificado os seguintes aspectos como prioritários nesse contexto (BUNDESMINISTERIUM FÜR WIRTSCHAFT UND TECHNOLOGIE, 2006):

a) suporte de inovativas PME's orientadas à tecnologia;

b) suporte de esforços que interliguem pesquisas e negócios; e

c) suporte de projetos inovativos, em particular aqueles com grande impacto em todos os setores ou na economia como um todo.

Depois de apresentados o contexto histórico no qual a Alemanha se fundamenta para promover suas redes de cooperação, a atual política de promoção de PME's por meio de redes de cooperação voltadas para inovação, e a iniciativa em nível nacional de redes de competência, o tópico a seguir visa apresentar como esse sistema de desenvolvimento local funciona em nível regional, explorando o caso da região de Berlim-Brandemburgo.

\subsection{Caso de desenvolvimento regional - Berlim-Brandemburgo}

Berlim é a capital alemã e um dos dezesseis estados nacionais. É a maior cidade do país, com uma população de 3,4 milhões de pessoas e apresenta um Produto Interno Bruto de 78 bilhões de euros, dos quais 4,2\% (mais de 3 bilhões) são destinados em pesquisa e educação. Brandemburgo, que é o Estado que circunda a capital Berlim, possui 2,5 milhões de pessoas e um PIB

Tabela 2. Critérios de admissão - Kompetenznetze Deutschland. Fonte: Adaptado de Velling (2006).

\begin{tabular}{cl}
\hline Critérios & Descrição dos critérios de admissão - Kompetenznetze.de \\
\hline 1 & A rede precisa apresentar um foco temático, uma especificidade. \\
2 & É requerida uma concentração e ancoragem regional, embora a atuação possa ser global. \\
3 & Espera-se uma organização e identidade da rede: \\
& - ativas e duráveis cooperações entre os atores na rede; \\
& - clara visibilidade para o mercado (por exemplo, website conjunto, materiais impressos, etc.); e \\
& - abertura para novos membros. \\
& Participação de vários atores com diferentes níveis de agregação de valor: \\
& - universidades, faculdades e instalações de pesquisa; \\
& - empresas (grandes, PME’s e novas empresas); \\
& - setor de serviços, especialmente na área financeira; \\
& - educação e formação superior; \\
& - pelo menos uma universidade/centro de pesquisa e papel ativo das empresas; e \\
& - pelo menos 10 atores, dentre os quais, no mínimo, 50\% representado por empresas. \\
& Desenvolvimento colaborativo de tecnologia: \\
& - qualidade e intensidade do trabalho em cooperação; e \\
& - indicadores - patentes em conjunto, freqüência de cooperação, etc. \\
& Sustentabilidade da rede de cooperação: \\
& - pelos menos 2 anos de existência (regra geral); \\
& - de base contínua (não apenas um projeto); \\
& - existência de firmes financiamentos (contribuição dos membros, patrocinadores, etc); e \\
& - por meio de suporte público: mas com estratégia sustentável para continuidade após término do apoio. \\
& A rede deve apresentar potencial para inovação e agregação de valor. \\
&
\end{tabular}




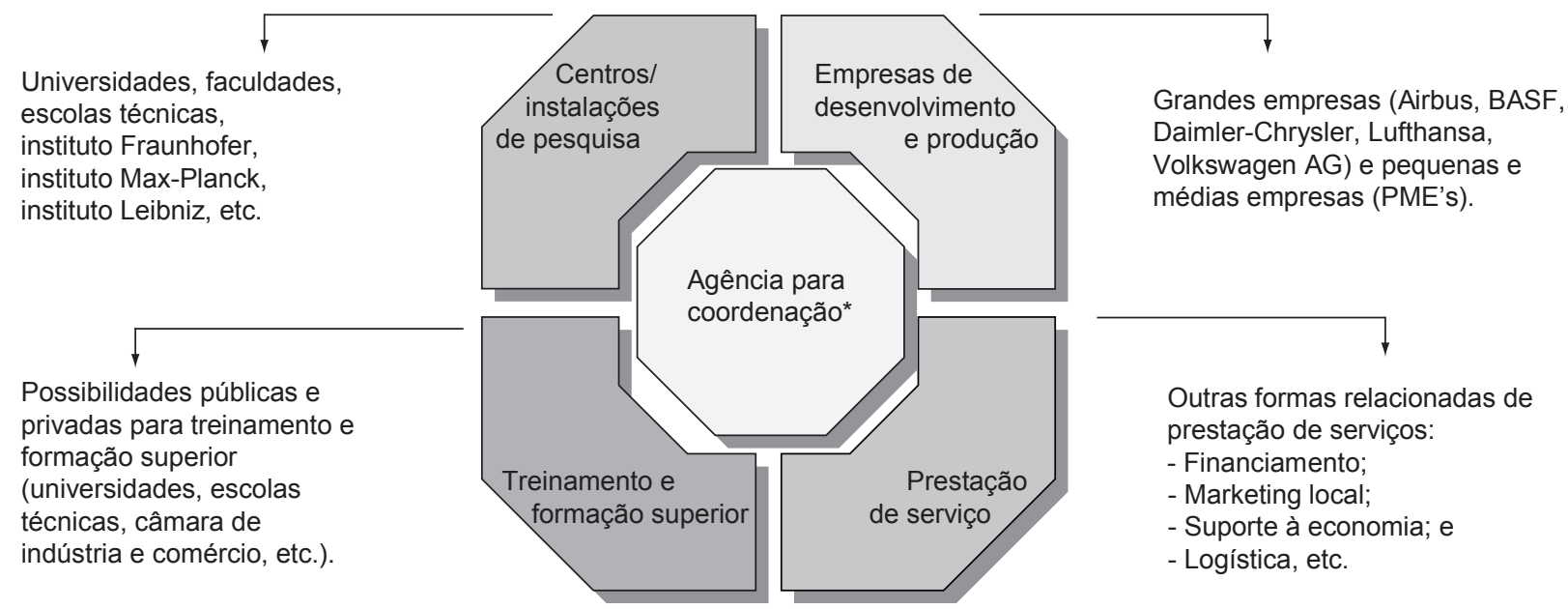

Figura 2. Estrutura ideal de uma rede de competência (VELLING, 2006; ESTERMANN, 2006). * Coordenação e governança operacional da rede de competência (gerente, coach, broker, auditor).

de 48 bilhões de euros. Durante quarenta anos Berlim foi uma cidade dividida, mas depois da queda do muro em 1989 e da reunificação alemã em 1990, foi constatado o enfraquecimento econômico de Berlim e de toda região pertencente a então Alemanha Oriental, incluindo, além de Berlim, os Estados de Brandenburg, MecklemburgVorpommern, Sachsen, Sachsen-Anhalt e Thüringen. Os efeitos causados pelo sistema socialista puderam ser percebidos em termos de elevada perda de empregos e conseqüente alta taxa de desemprego, permanência de poucas grandes companhias e milhares de pequenas e médias empresas envolvidas num contexto de crise econômica. Por outro lado, Berlim apresentava um enorme potencial científico e cultural (BROICH, 2006).

Atualmente a cidade de Berlim e sua região metropolitana, que já envolve o estado de Brandemburgo, conta com quatro grandes Universidades (Technische Universität Berlin, Frei Universität Berlin, Humboldt Universität Berlin e Universität Potsdam), sete universidades de ciências aplicadas, 70 institutos de pesquisa, 89 clínicas e hospitais, seis grandes parques tecnológicos e um total de 150.000 estudantes. Uma das estratégias de desenvolvimento regional é a busca pela concentração de indústrias de alta tecnologia e inovação em específicos campos de competência (ou centros de excelência) regionais, conforme destaca a Tabela 3.

De acordo com a TSB (TECHNOLOGIESTIFTUNG BERLIN, 2005), os centros de excelência em Berlim consistem de todas as organizações científicas e econômicas, e todas as instituições, iniciativas, redes, associações e agentes que sejam relevantes em um determinado campo de tecnologia. Ainda, tal campo deve demonstrar alto potencial científico e econômico na região metropolitana de Berlim-Brandemburgo e poder, portanto, promover as condições adequadas para o crescimento sustentável.

Broich (2006) retrata que as funções e tarefas dentro de um contexto que favoreça estratégias de inovação são realizadas por quatro principais atores: poder público, fundação de tecnologia, parceiros locais e banco de investimento de Berlin. Esse conjunto de atores é denominado Organização Quadriga. Dentro dessa abordagem, os participantes desenvolvem em conjunto planos mestres para cada centro de excelência. Os planos mestres detalham objetivos estratégicos, os planos de ações e o nome do Parceiro Quadriga que é responsável pelo planejamento e implementação das ações. Também é de fundamental importância nesse processo de desenvolvimento local o papel das instituições de pesquisa e empresas como alavancas à inovação e campos regionais de competência, além do papel estratégico do poder público no estabelecimento de diretrizes para o desenvolvimento regional.

Dessa forma, o Estado persegue o objetivo de desenvolver centros de excelência em um contexto de rápido crescimento, internacionalmente competitivo, e oferecendo suporte mútuo para clusters de ciência e negócios, com uma forte dinâmica em inovação. Os instrumentos para se atingir essas metas são: o suporte para tecnologia e inovação, o estímulo para negócios e um conjunto de recursos e atividades. A Figura 3 ilustra como a TSB entende o processo de inovação objetivando o desenvolvimento de clusters, por exemplo, na área de saúde, na região Berlim-Brandemburgo, em função de centros de excelência derivados, por sua vez, de campos de inovação e áreas tecnológicas (TECHNOLOGIESTIFTUNG BERLIN, 2005).

Finalmente, de acordo com essa linha de desenvolvimento de clusters, a visão estabelecida para o desenvolvimento econômico de Berlim e Brandemburgo, 
Tabela 3. Redes de competência na região de Berlim-Brandemburgo. (BUNDESMINISTERIUM FÜR WIRTSCHAFT UND TECHNOLOGIE, 2006; TECHNOLOGIESTIFTUNG BERLIN, 2005).

\begin{tabular}{|c|c|c|c|}
\hline Campos de inovação & Redes de competência & Região/abrangência & Iniciativa \\
\hline Biônica & BIOKON - Bionics Competence Network & Berlim-Brandemburgo & kompetenznetze.de \\
\hline Biotecnologia & $\begin{array}{l}\text { BioHyTec - Brandenburg-Berlin Biohybrid } \\
\text { Technologies Network }\end{array}$ & Berlim-Brandemburgo & kompetenznetze.de \\
\hline Biotecnologia & BioProfile Nutrigenomics Berlin-Brandenburg & Berlim-Brandemburgo & kompetenznetze.de \\
\hline Biotecnologia & BioTOP Berlin-Brandenburg & Berlim-Brandemburgo & TSB/kompetenznetze.de \\
\hline Biotecnologia & RiNA - RNA Technologies Network & Berlim-Brandemburgo & kompetenznetze.de \\
\hline Genoma & $\begin{array}{l}\text { GABI - Genome Analysis of the Plant Biological } \\
\text { System }\end{array}$ & Berlim-Brandemburgo & kompetenznetze.de \\
\hline $\begin{array}{l}\text { Eng. Mecatrônica e } \\
\text { Microssistemas }\end{array}$ & Centre for Microsystems Technology (ZEMI) & Berlim-Brandemburgo & kompetenznetze.de \\
\hline Eng./Tec. Médica & TSBmedici - Center of Medical Technology Berlin & Berlim / Nacional & TSB \\
\hline Medicina & Competence Network for Congenital Heart Defects & Berlim / Nacional & kompetenznetze.de \\
\hline Medicina & Competence Network Stroke & Berlim / Nacional & kompetenznetze.de \\
\hline Medicina & Heart Failure Competence Network & Berlim / Nacional & kompetenznetze.de \\
\hline Medicina & $\begin{array}{l}\text { Medical Research Network Pediatric Oncology and } \\
\text { Hematology }\end{array}$ & Berlim / Nacional & kompetenznetze.de \\
\hline Nanotecnologia & $\begin{array}{l}\text { NanoOp - Competence Center for the Application of } \\
\text { Nanostructures in Optoeletronics }\end{array}$ & Berlim-Brandemburgo & kompetenznetze.de \\
\hline $\begin{array}{l}\text { Tec. Informação e } \\
\text { Comunicação (ICT)/Mídia }\end{array}$ & $\begin{array}{l}\text { MO-SIS Mobile Security Systems/NEST Network for } \\
\text { Integ. Systems in Telemedicine (TimeKontor) }\end{array}$ & Berlim-Brandemburgo & TSB \\
\hline Tec. Ópticas/Tec. em Laser & OpTec-Berlin-Brandenburg $(O p T e c B B)$ e.V. & Berlim-Brandemburgo & TSB/kompetenznetze.de \\
\hline Transporte e Mobilidade & FAV/TSB Transport Technologies Berlin & Berlim-Brandemburgo & TSB/kompetenznetze.de \\
\hline
\end{tabular}

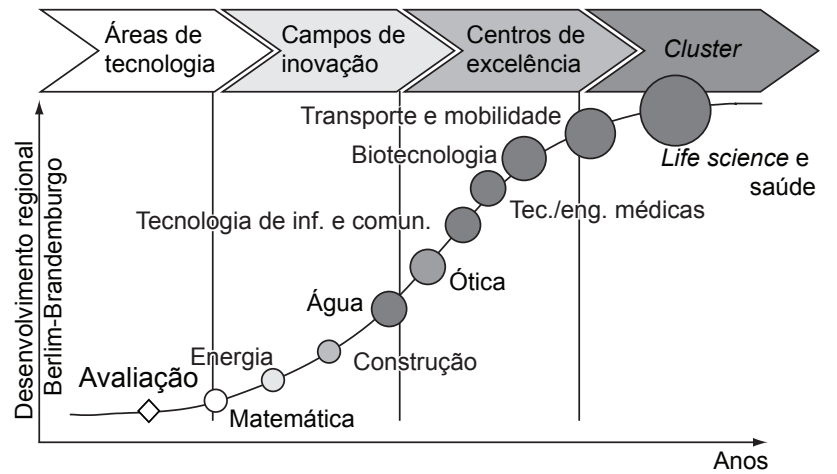

Figura 3. Processo contínuo de inovação Berlim-Brandemburgo (TECHNOLOGIESTIFTUNG BERLIN, 2005, p. 6).

em função dos atuais centros de excelência, está embasada na concentração de três principais clusters até 2015 nas seguintes áreas temáticas (BROICH; MICHL, 2006): i) saúde; ii) cultura, tecnologia de informação e comunicação (ICT), multimídia; e iii) mobilidade e transporte.

\section{Propostas para o fortalecimento de clusters e redes de cooperação no Brasil}

No aspecto europeu, os clusters e redes de cooperação têm apresentado interessantes resultados em termos de inovação, desempenho e competitividade e podem servir como fontes de benchmarking para o desenvolvimento de tais práticas no Brasil. Para se atingir resultados posi- tivos, entende-se que as políticas de apoio aos clusters e redes de cooperação devem ser direcionadas para as especificidades de cada região e devem buscar minar as potenciais barreiras ao seu desenvolvimento. As autoridades públicas devem atuar como facilitadoras no processo de cooperação, oferecendo infra-estrutura e dando suporte ao surgimento de instituições formais que tenham capacidade de coordenar as atividades do cluster. O foco então deve ser para integração com universidades e centros de pesquisa, estimulando o empreendedorismo e a inovação, conforme ilustrou o caso alemão.

Quanto às atuais políticas de investimento em clusters $\mathrm{e}$ redes de cooperação na Europa, a inovação tem aparecido como elemento estratégico. Analisando em profundidade o caso alemão e suas estratégias de implementação, fica evidente a existência de uma base sólida que permite o desenvolvimento acelerado de redes de cooperação e clusters. A Alemanha apresenta um passado de investimentos em vários fatores que permitiram, mesmo depois de amplamente afetada por duas guerras, seguir entre as cinco maiores economias mundiais. Apesar de focar pouco no desenvolvimento regional até o início da década de 90, a Alemanha traçou uma estratégia de desenvolvimento de novas tecnologias a partir do apoio à formação de redes regionais de competência estimulando a integração entre PME's, centros de pesquisa, universidades, centros de educação e treinamento, e contando com o apoio do poder público em níveis estadual e federal. 
Entretanto, o funcionamento e interação devem ser de responsabilidade de agências locais de coordenação para cada rede, ou seja, entidades formais que não apenas enxerguem as economias externas como algo benéfico, mas também busquem trazer à rede projetos conjuntos que agreguem valor e propiciem a eficiência coletiva por meio de coordenação, cooperação e inovação.

O caso alemão de desenvolvimento de clusters e redes de cooperação de PME's com foco em inovação, em particular a iniciativa Kompetenznetze Deutschland, apresenta peculiaridades que podem ser destacadas e adaptadas à realidade brasileira. Por um lado, a Alemanha está entre as cinco economias mais fortes do mundo e representa a principal força econômica da Europa, o que significa mais chance de sucesso em sua atual estratégia de desenvolvimento de redes de cooperação e clusters de PME's. Nesse aspecto é difícil adaptar programas para o contexto brasileiro em função de inúmeras diferenças. Por outro lado, as regiões onde se encontram os novos Estados alemães apresentavam-se ao final do regime socialista de1989 muito frágeis economicamente e, apesar de ainda apresentarem alto índice de desemprego em relação à média alemã, vêm mostrando rápida evolução, principalmente no que se refere à pesquisa e inovação, usando para tal a estratégia de promoção de clusters e redes de cooperação como alavanca para o desenvolvimento econômico.

A análise específica da região de Berlim-Brandemburgo sugere algumas lições interessantes e pode ser usada como benchmarking para políticas de desenvolvimento de clusters de base tecnológica no Brasil, especificamente nas regiões Sul e Sudeste onde se estabelecem em sua maioria. São Paulo, que apresenta a maior incidência de clusters de base tecnológica, poderia usar os resultados aqui descritos para guiar a sua atual política de desenvolvimento de parques tecnológicos, buscando um modelo de gestão adequado para os pólos de São José dos Campos, Campinas, São Carlos, Ribeirão Preto e Grande São Paulo. O caso específico da cidade de São Carlos é um interessante exemplo. Comparando-o com o caso apresentado de Berlim, a integração entre governos estadual e municipal, universidades, empresas (principalmente PME's) e centros de pesquisa poderia possibilitar uma infra-estrutura de suporte para gestão de redes de cooperação que, por sua vez, teriam condições de impulsionar o desenvolvimento do parque tecnológico de São Carlos para tornar-se um sistema regional de inovação de alta tecnologia best practice no Brasil.

Como importantes experiências observadas no observatório europeu, bem como na análise das redes de competência alemãs e o caso específico da região de Berlim-Brandemburgo, podem ser estabelecidas possíveis diretrizes para auxiliar o Governo do Estado de São Paulo e demais Governos de outros Estados brasi- leiros no seu processo de desenvolvimento de clusters de alta tecnologia e arranjos produtivos locais:

a) governos Estaduais e Federal deveriam assumir um papel mais próximo, não apenas indicando a importância dos clusters, mas sim oferecendo infraestrutura e instrumentos iniciais para promover a rede de cooperação. Além disso, o apoio público poderia estar atrelado à abertura de financiamento concedido por meio de competição para formação de redes de cooperação, respeitando critérios como os definidos pela iniciativa alemã;

b) deve ser estimulado o foco em inovação. Como o Brasil apresenta em sua maioria clusters tradicionais, é importante que eles sejam incentivados a inovar, principalmente em produtos/processos e novos métodos para marketing, acesso a mercado e distribuição. Além disso, como estratégia nacional, é importante incentivar o fortalecimento de redes de cooperação de base tecnológica, preferencialmente próximas a centros de pesquisa de alta tecnologia, como já ocorre em várias regiões do Brasil; e

c) o processo de gestão de redes precisa ser mais bem definido. É preciso definir estruturas e infra-estruturas de apoio. Quais processos são necessários para se gerenciar uma rede de cooperação? Quais profissionais, funções e respectivas competências devem ser alocadas (gerente, coach, broker, auditor)? Como se mede a melhoria de desempenho da rede (indicadores) e como mostrar esse impacto em níveis setoriais ou nacionais?

\section{Considerações finais}

Este trabalho apresentou um relato sobre o processo de desenvolvimento de clusters e redes de cooperação na Europa, analisando os estudos da Comissão Européia e uma descrição do caso alemão de incentivo a redes de cooperação e clusters regionais por meio do programa Kompetenznetze Deutschland, abordando mais detalhadamente a estratégia de desenvolvimento de clusters na região de Berlim-Brandemburgo.

Embora as políticas de desenvolvimento de clusters e as redes de cooperação européias apresentem resultados interessantes, tal estratégia não deve ser usada como uma panacéia para os problemas econômicos de diferentes regiões e países. Ainda que a Europa precise realizar análises mais apuradas de seus clusters e que a comparação entre diferentes clusters seja uma tarefa difícil, principalmente entre países geograficamente mais distantes como Alemanha e Brasil, os resultados mostrados pelos estudos dos clusters europeus e pelas redes de competência alemãs sugerem que esforços para troca de informações e benchmarking poderiam auxiliar o processo de desenvolvimento de clusters e redes 
de cooperação em países em desenvolvimento, como é o caso do Brasil.

Complementarmente, as atividades realizadas pelos governos federal e estaduais para promover clusters e redes regionais de cooperação deveriam também considerar a promoção de desenvolvimento regional sustentável. Para tanto, áreas estratégicas de investimento deveriam ser escolhidas, por exemplo, investimento em clusters ou redes de cooperação focados em inovação, e então estabelecer parcerias com governos locais e atender às estruturas e infra-estruturas necessárias para o desenvolvimento de agências regionais de desenvolvimento ou institutional thickness. A integração entre tais agências regionais, centros de pesquisa, universidades, escolas técnicas, pequenas, médias e grandes empresas, instituições de crédito, e outros tipos de stakeholders locais deveria ser incentivada. Uma agenda para desenvolvimento sustentável poderia ser planejada entre tais stakeholders por meio de ações locais conjuntas, que deveriam ser focadas em inovação, desempenho e competitividade, e ainda consider a perspectiva social e a ambiental de desenvolvimento sustentável. Dessa forma, nós entendemos que alguns elementos-chave poderiam ser aqui destacados como essenciais para esse processo de integração, o qual nós chamaríamos de gestão do processo de desempenho e inovação em clusters e redes regionais de cooperação, que abrange os seguintes passos: a) identificação de stakeholders, bem como suas necessidades e contribuições;

b) definição de objetivos e estratégias comuns;

c) implementação de ações de melhoria por meio de projetos conjuntos focados em melhorias e inovações em gestão, tecnologias, processos, produtos e serviços;

d) desenvolvimento de um sistema de avaliação e medição de desempenho como, por exemplo, um balanced scorecard;

e) fortalecimento do capital social; e

f) uma infra-estrutura de suporte ao processo de gestão.

Finalmente, é importante também observar a importância das autoridades públicas, principalmente nos primeiros anos, no processo de desenvolvimento regional por meio de diretrizes estratégicas e investimentos em educação, pesquisa, projetos e infra-estrutura de gestão. A participação de organizações não governamentais para a promoção de clusters e redes de cooperação também deve ser mais bem compreendida. As redes de cooperação, por sua vez, deveriam oferecer feedback constante para as autoridades públicas e seus stakeholders sobre o desempenho em termos de objetivos estratégicos, inovação, competitividade e crescimento sustentável.

\title{
Clusters and cooperation networks of small and medium-sized companies: analysis of european and German experiences and contributions to the brazilian case
}

\begin{abstract}
This paper presents studies on clusters and cooperation networks of small and medium-sized enterprises (SMEs) developed by the European Commission, as well as a description of the current German strategy for developing competence networks (Kompetenznetze Deutschland), focusing specifically on the case of the regions of Berlin and Brandenburg. The main goal of this work is to analyze the experience of the European and German initiatives and to verify the possibilities of adapting such initiatives to the context of local productive systems and technology parks in Brazil. In general, the SMEs in European clusters were found to perform better than other SMEs in their respective sectors. Increasing competitiveness of clusters and regional economic growth were found to be strongly tied to investments in innovation. The lessons learned from this study which could possibly be applied to Brazil are a more direct support of the public sector, a stronger focus on innovation, and the definition of management processes for cooperation networks.
\end{abstract}

Keywords: Germany. Brazil. Clusters. Competence networks. Cooperation networks. 
ANDRIANI, P. et al. Challenging Clusters. The Prospects and Pitfalls of Clustering for Innovation and Economic Development. Summary Report from an AIM Management Research Forum in Cooperation With the Welsh Economy Research Unit. Advanced Institute of Management Research, jun. 2005.

BROICH, B. Combining National and Community Funding: Experience and Prospects from Berlin. In: REGIONS FOR ECONOMIC CHANGE - INNOVATING THROUGH EU REGIONAL POLICY. Brussels, 12-13 jun. 2006. Disponível em: <http://ec.europa.eu/regional_policy/conferences/ innovating_june06/doc/w3/wks3c_broich.ppt> Acesso em: 3 jan. 2007.

BROICH, B.; Michl, B. Regional Innovation System Berlin and the EURO-COOP Project. In: EURO-COOP THIRD REGIONAL WORKSHOP. Paris, 4-5, dez. 2006. Disponível em: <http://www.iccr-international.org/euro-coop/workshops/ regional-ws3.html >. Acesso em: 3 jan. 2007.

BMBF - BUNDESMINISTERIUM FÜR BILDUNG UND FORSCHUNG. Erfahrungen aus EXIST - Querschau über die einzelnen Projekte. BMBF Studie, Referat Öffentlichkeitarbeit, Bonn, Jun. 2005. Disponível em: <http://www.bmbf.de/pub/ erfahrungen_aus_exist-querschau.pdf $>$. Acesso em: 9 jan. 2007.

BMBF - BUNDESMINISTERIUM FÜR BILDUNG UND FORSCHUNG. Unternehmen Region: Die BMBFInnovationsinitiative Neue Länder. Referat Publikationen: Internetredaktion, Bonn, Berlin, 2004. Disponível em: http:// www.bmbf.de/pub/unternehmen_regionen.pdf. Acesso em: 9 jan. 2007.

BMBF - BUNDESMINISTERIUM FÜR BILDUNG UND FORSCHUNG. BioRegionen in Deutschland: starke Impulse für die nationale Technologieentwicklung. Referat Publikationen: Internetredaktion, 3. überarbeitete Auflage, Bonn, Berlin, 2005. Disponível em: <http://www.bmbf.de/pub/ bioregionen_in_deutschland.pdf>. Acesso em: 9 jan. 2007.

BMWI - BUNDESMINISTERIUM FÜR WIRTSCHAFT UND TECHNOLOGIE. Kompetenznetze.de 2006/2007 - networking for innovation. BMWi, Referat P3. Berlin, April, 2006. Disponível em: <http://www.kompetenznetze. de/Redaktion/Zentralredaktion/Bestellungen/medien/ Jahresbericht-2006-2007-pdf,property=pdf.pdf $>$. Acesso em: 17 jul. 2006.

CARVALHO, M. M.; MACHADO, S. A.; RABECHINI Jr., R. Fatores Críticos de Sucesso em Empresas de Base Tecnológica. Revista Produto \& Produção, v. 4, número especial, p. 47-59, abr. 2000 .

CHANDLER Jr., A. D. Scale and Scope: The Dynamics of Industrial Capitalism. Cambridge, Massachusetts: Harvard University Press, 1990.

ESTERMANN, R. Mehr Mehrwert mit Kompetenznetze Deutschland. In: JAHRESTAGUNG KOMPETENZNETZE DEUTSCHLAN. 2006. Disponível em: <http://www. kompetenznetze.de/navi/de/root,did=153320.html>. Acesso em: 12 dez. 2006.

EUROPEAN COMMISSION. Observatory of European SMEs 2002. SME in focus: Main results from the 2002 Observatory of European SMEs. 2002 a. Disponível em: <http://ec.europa. eu/enterprise/enterprise_policy/analysis/doc/execsum_2002_ en.pdf>. Acesso em: 6 dez. 2006.

EUROPEAN COMMISSION. Observatory of European SMEs 2002, No. 3 - Regional clusters in Europe. Report submitted to the Enterprise Directorate General by KPMG Special Services, EIMB Business \& Policy Research, and ENSR. Luxembourg: Office for Official Publications of the European Communities, 2002 b. Disponível em: <http://ec.europa.eu/enterprise/ enterprise_policy/analysis/doc/smes_observatory_2002_ report3_en.pdf $>$. Acesso em 17 ago. 2006.

EUROPEAN COMMISSION. Final Report of the Expert Group on Enterprise Cluster and Networks. European Commission Enterprise Directorate-General. 2002 c. Disponível em: <http:// ec.europa.eu/enterprise/entrepreneurship/support_measures/ cluster/final_report_clusters_en.pdf. / http://ec.europa.eu/ enterprise/entrepreneurship/support_measures/cluster/map_ project.htm>. Acesso em: 2 jun. 2006.

EUROPEAN COMMISSION. Observatory of European SMEs 2003, No. 5 - SMEs and cooperation. 2004 a. Disponível em: <http://ec.europa.eu/enterprise/enterprise_policy/analysis/ observatory _en.htm>. Acesso em: 30 out. 2006.

EUROPEAN COMMISSION. Observatory of European SMEs 2003, No. 8 - Highlights from the 2003 Observatory. 2004 b. Disponível em: <http://ec.europa.eu/enterprise/enterprise_ policy/analysis/ observatory_en.htm>. Acesso em: 30 out. 2006.

KETELS, C. Clusters of Innovation in Europe, in Structural Change in Europe 3 - Innovative City and Business Regions. Bollschweil: Hagbarth Publications, 2004. Disponível em: $<$ http://www.isc.hbs.edu/pdf/Ketels_European_Clusters_2004. pdf>. Acesso em: 17 ago. 2006.

KRUGMAN, P. Geography and Trade. Cambridge: MIT Press, 1991.

KOMPETENZNETZE DEUTSCHLAND: NETWORKING FOR INNOVATION. Disponível em: <http://www.kompetenznetze. de/>, 2008.

MINISTÉRIO DO AMBIENTE. Contributos para uma Política de Reforço da Atractividade e Dinamismo Económico dos Eixos Urbanos Não Metropolitanos - Estudo de Casos Internacionais. Lisboa: Departamento de Prospectiva e Planejamento (com a participação da União Européia - projeto cofinanciado pelo FEDER), 30 de mar. 2006. (Relatório elaborado ao abrigo de protocolo estabelecido em 20/02/06 entre o DPP e o Gestor da Iniciativa Comunitária Interreg III).

MINISTÉRIO DO DESENVOLVIMENTO, INDÚSTRIA E COMÉRCIO EXTERIOR. Arranjos Produtivos Locais (APLs). 2006. Disponível em: <http://www.desenvolvimento. gov.br/sitio/sdp/ proAcao/arrProLocais/arrProLocais.php $>$. Accesso em: 15 mai. 2006.

PORTER, M. E. Clusters and the new economics of competition. Harvard Business Review, New York, v. 76, n. 6, p. 77-90, nov./dec. 1998.

PUGA, F. Alternativas de apoio a MPMES localizadas em arranjos produtivos locais. 2003. Disponível em: <http:// redeincubar.anprotec.org.br:8280/portal/baixaFcdAnexo. do?id=6>. Acesso em: 12 nov. 2004 .

QUANDT, C. O. Inovação em Clusters Emergentes. ComCiência: Revista Eletrônica de Jornalismo Científico - Desafios da Inovação, n. 57, p.1-5. ago. 2004. Disponível em: <http://www. comciencia.br/reportagens/2004/08/13.shtml>. Acesso em: 17 jan. 2007.

SCHMITZ, H. Collective efficiency and increasing return. Cambridge Journal of Economics, v. 23, n. 4, p. 465-483, jul 1999. Disponível em: <http://proquest.umi.pwdweb>. Acesso em: 27 ago. 2004. 
SCHMITZ, H.; NADVI, K. Clustering and industrialization: introduction. World Development, v. 27, n. 9, p. 1503-1514, 1999.

SEBRAE. Boletim Estatístico de Micro e Pequenas Empresas. Observatório SEBRAE, $1^{\circ}$ semestre, 2005. Disponível em: $<$ http://www.sebrae.com.br/br/mpe\%5Fnumeros/>. Acesso em: 24 abr. 2006.

TECHNOLOGIESTIFTUNG BERLIN. Jahresbericht 2005. Berlin: TSB Technologie Stiftung Berlin für Wirtschaft + Innovation, 2005.
VAN DER LINDE, C. The Demography of Clusters. Findings from the Cluster Meta-Study. In: Bröcker, J.; Dohse, D.; Soltwedel, R. (Eds.) Innovation Clusters and Interregional Competition. Berlin, Heidelberg, New York: Springer, 2003, p. $130-149$.

VELLING, J. Strategische Ausrichtung der Initiative Kompetenznetze Deutschland im BMWi. In: Jahrestagung Kompetenznetze Deutschland. Berlim, 2006. Disponível em: <http://www.kompetenznetze.de/navi/de/root,did=153320. html>. Acesso em: 12 dez. 2006.

\section{Sobre os autores}

\section{Mateus Cecílio Gerolamo}

Departamento de Engenharia de Produção, Escola de Engenharia de São Carlos, Universidade de São Paulo, USP, Avenida Trabalhador São-carlense, 400, Centro, CEP 3566-590, São Carlos, SP, Brasil,

Departamento de Tecnologia de Montagem e Gestão da Fábrica, Instituto de Máquinas-Ferramentas e Gestão da Fábrica, Universidade Técnica de Berlim, Pascalstrasse 8-9, D-10587, Berlim, Alemanha, e-mail: mateus.gerolamo@gmail.com

\section{Luiz César Ribeiro Carpinetti}

Departamento de Engenharia de Produção, Escola de Engenharia de São Carlos, Universidade de São Paulo, USP, Avenida Trabalhador São-carlense, 400, Centro, CEP, 3566-590, São Carlos, SP, Brasil, e-mail: carpinet@sc.usp.br

\section{Timo Fleschutz}

\section{Günther Seliger}

Departamento de Tecnologia de Montagem e Gestão da Fábrica, Instituto de Máquinas-Ferramentas e Gestão da Fábrica, Universidade Técnica Berlim,Pascalstrasse 8-9, D-10587, Berlim, Alemanha,

e-mails: timo.fleschutz@mf.tu-berlin.de; guenther.seliger@mf.tu-berlin.de

Agradecimentos: À Coordenação de Aperfeiçoamento de Pessoal de Nível Superior (CAPES) e ao Serviço Alemão de Intercâmbio Acadêmico (DAAD), pelo financiamento concedido à pesquisa.

Ao professor Jörg Sydow, Frei Universität Berlin/Chairman of kompetenznetze.de Advisory Council e ao Dr. Bruno Broich CEO Technologiestiftung Berlin (TSB), pela disponibilidade em fornecer valiosas informações sobre as redes de cooperação em Berlim e Brandemburgo. Aos revisores da revista Gestão e Produção, pelos comentários que contribuíram para a qualidade do artigo. 